\title{
Attending to risk in sequential sampling plans
}

\author{
A. J. Hamilton ${ }^{1,2}$, V. L. Versace ${ }^{1}$, G. Hepworth ${ }^{3}$, F. Stagnitti ${ }^{1}$, \\ J. Dawson ${ }^{2}$, P. M. Ridland ${ }^{2}$, N. M. Endersby ${ }^{2,4}$, N. A. Schellhorn ${ }^{5}$, \\ C. Mansfield ${ }^{2} \&$ P. M. Rogers ${ }^{2}$ \\ ${ }^{I}$ School of Ecology and Environment, Deakin University, Australia \\ ${ }^{2}$ Department of Primary Industries, Victoria, Australia \\ ${ }^{3}$ Department of Mathematics \& Statistics, The University of Melbourne, \\ Australia \\ ${ }^{4}$ CESAR Centre for Environmental Stress and Adaptation Research, \\ School of Biological Sciences, Monash University, Australia \\ ${ }^{5}$ CSIRO Entomology, Australia
}

\begin{abstract}
Researchers typically tackle questions by constructing powerful, highlyreplicated sampling protocols or experimental designs. Such approaches often demand large samples sizes and are usually only conducted on a once-off basis. In contrast, many industries need to continually monitor phenomena such as equipment reliability, water quality, or the abundance of a pest. In such instances, costs and time inherent in sampling preclude the use of highlyintensive methods. Ideally, one wants to collect the absolute minimum number of samples needed to make an appropriate decision. Sequential sampling, wherein the sample size is a function of the results of the sampling process itself, offers a practicable solution. But smaller sample sizes equate to less knowledge about the population, and thus an increased risk of making an incorrect management decision. There are various statistical techniques to account for and measure risk in sequential sampling plans. We illustrate these methods and assess them using examples relating to the management of arthropod pests in commercial crops, but they can be applied to any situation where sequential sampling is used.
\end{abstract}

Keywords: binomial, enumerative, risk, sampling plan, sequential sampling, Taylor's power law, Wald's sequential probability ratio test. 


\section{Introduction: constructing sequential sampling plans}

Sequential sampling is popular in applied disciplines such as agricultural entomology as it demands the collection of the minimum number of samples necessary to make a pest management decision. Surveying crops is timeconsuming and expensive, and growers cannot afford the luxury of a highlyintensive sampling programme. In sequential sampling, surveying continues until a predefined stop-rule is satisfied. That is, the ultimate sample size is determined by the results obtained as sampling progresses.

The two most commonly-used types of sequential sampling plans are the enumerative and the binomial. In the context of applied entomology, enumerative plans involve counting individual insects so as to obtain an estimate of the population's density. This estimate is then compared to an action threshold (AT). The AT represents the population density above which the grower will employ a control measure, such as pesticide application. In contrast, binomial plans only require one to record whether or not the number of insects on each sampling unit exceeds a predefined tally threshold (usually zero, hence the alternative name presence-absence sampling, Hepworth and McFarlane $[1,2])$. The stop-rule incorporates the AT, and upon cessation of sampling the user is informed as to whether or not action is required.

\subsection{Enumerative sampling plans}

As explained by Karandinos [3], the minimum sample size $\left(n_{\min }\right)$ required for a survey can be adequately approximated as

$$
n_{\min }=s^{2} /(D \bar{x})^{2}
$$

where $s^{2}$ and $\bar{x}$ are the sample variance and sample mean respectively, and $D$ is the nominal, i.e. desired, level of precision (expressed as $\sigma_{\bar{x}} / \bar{x}$, where $\sigma_{\bar{x}}$ is the standard error of the mean). Prior to sampling, however, we do not know $\bar{x}, s^{2}$, or $\sigma_{\bar{x}}$, so we cannot determine $n_{\min }$. A possible solution would be to conduct a pilot survey before each sampling event so that these parameters could be estimated. This would plainly be impractical for routine sampling programmes. The problem can be resolved through the construction of a sequential sampling stop-boundary. Such a boundary is a function of the variance-mean relation of the pest (in effect, its spatial distribution) and the desired level of precision of the survey. Two stop boundaries have been proposed: Kuno [4] and Green [5].

The foundation of Kuno's [4] approach lies in the use of Iwao's [6] patchiness regression technique to characterise the variance-mean relation. Iwao modelled the relation between the sample mean $(\bar{x})$ and Lloyd's [7] mean crowding index $\left(x^{*}\right)$, which is calculated as

$$
x^{*}=\bar{x}+\left[\left(s^{2} / \bar{x}\right)-1\right]
$$


Iwao's patchiness regression, whereby $x^{*}$ is regressed against $\bar{x}$, is thus

$$
x^{*}=\alpha+\beta \bar{x}
$$

where the $y$-intercept $(\alpha)$ is the index of basic contagion and the slope $(\beta)$ is the density-contagiousness coefficient. Finally, the minimum sample size for a prescribed level of precision can be calculated as

$$
n=[(\alpha+1) / \bar{x}+(\beta-1)] / D^{2}
$$

Iwao and Kuno [8]. Having obtained $n$, Kuno's stop boundary can be calculated:

$$
T_{n[\text { Kuno }]}=(\alpha+1) /\left(D^{2}-[(\beta-1) / n]\right)
$$

where $T_{n[\text { Kuno }]}$ is the cumulative number of individuals in samples 1 to $n$. Finally, a sequential stop-chart is constructed by plotting $T_{n[\text { Kuno] }}$ against $n$.

Green's [3] stop boundary is analogous to Kuno's, the major difference being that it uses Taylor's Power Law (TPL) Taylor [9] to model the variance-mean relation. TPL is

$$
s^{2}=a \bar{x}^{b}
$$

where $s^{2}$ and $\bar{x}$ are as described earlier, $a$ is a scaling factor that is dependent on sampling method and habitat, and the exponent $b$ is a measure of spatial contagion. The parameters $a$ and $b$ can be estimated (i.e. $\hat{a}$ and $\hat{b}$ ) by regressing $\ln s^{2}$ against $\ln \bar{x}$ :

$$
\ln s^{2}=\ln \hat{a}+\hat{b} \ln \bar{x}
$$

Thus, replacing $s^{2}$ in eqn. (1) with $\hat{a} \bar{x}^{\hat{b}}$ from eqns (5) and (6) we get

$$
n=\hat{a} \bar{x}^{(\hat{b}-2)} / D^{2}
$$

Green's [5] stop boundary can then be calculated as follows:

$$
T_{n[\text { Green }]}=\left(\frac{D^{2}}{\hat{a}}\right)^{1 /(\hat{b}-2)} n^{(\hat{b}-1) /(\hat{b}-2)}
$$




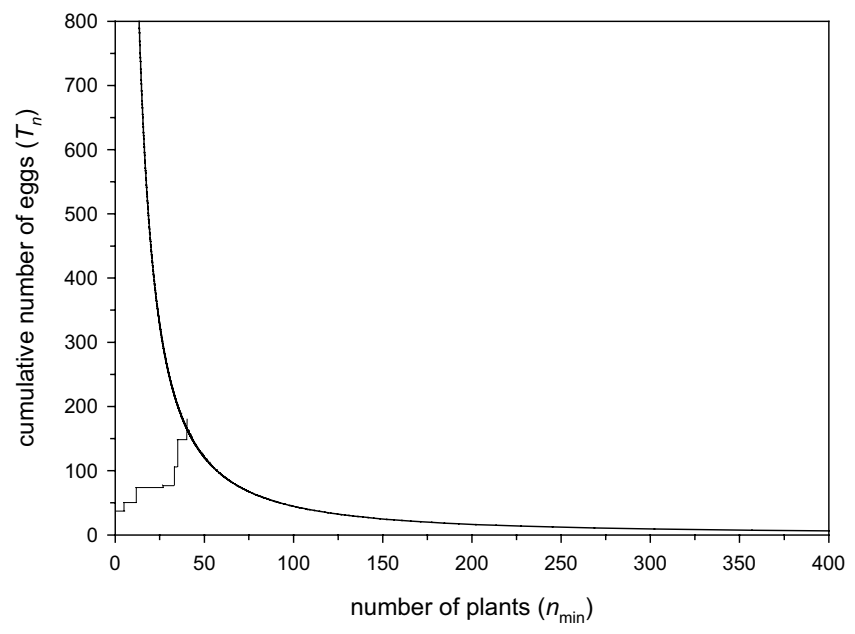

Figure 1: An enumerative sequential sampling chart for Helicoverpa spp. on fresh-market tomatoes (based on unpublished data of J.D. \& C.M.).

Having obtained $T_{n \text { [Green] }}$ it can be plotted against $n_{\min }$ to create a sequential sampling stop chart, as shown in fig. 1. To use such a chart, or an analogous chart derived from Kuno's method, one keeps track of the number of sampling units (plants in fig. 1) and the cumulative number of individuals (eggs in fig. 1) found. Sampling ceases when the line defining this relationship intersects the stop boundary. In fig. 1. the stepped-line is a hypothetical example to demonstrate how such a chart is used. In this particular scenario, 40 plants were surveyed and a total of 180 eggs were recorded before sampling ceased.

\subsection{Binomial plans}

The most commonly used binomial sampling plan is Wald's [10] sequential probability ratio test (SPRT), and this is the only binomial plan considered here. The SPRT comprises lower and upper stop boundaries, and sampling continues as long as one is between the two boundaries. If the upper boundary is crossed, sampling ceases and the plan recommends implementing control. Conversely, if the lower boundary is intersected, sampling stops and no control is taken. The $y$ intercepts for the lower and upper stop boundaries, $h_{0}$ and $h_{1}$ respectively, are calculated as follows:

$$
h_{0}=\frac{\ln [(1-\beta) / \alpha]}{\ln \left[\frac{\theta_{1}\left(1-\theta_{0}\right)}{\theta_{0}\left(1-\theta_{1}\right)}\right]} \quad h_{1}=\frac{\ln [\beta /(1-\alpha)]}{\ln \left[\frac{\theta_{1}\left(1-\theta_{0}\right)}{\theta_{0}\left(1-\theta_{1}\right)}\right]}
$$

where $\theta_{0}$ and $\theta_{1}$ respectively represent the lower and upper bounds about the AT. In other words, $\theta_{0}$ is the threshold below which control is not required and $\theta_{1}$ is 
the threshold above which control would be instigated. The common slope, $\lambda$, for the two lines is given as

$$
\lambda=\frac{\ln \left[\left(1-\theta_{0}\right) /\left(1-\theta_{1}\right)\right]}{\ln \left[\frac{\theta_{1}\left(1-\theta_{0}\right)}{\theta_{0}\left(1-\theta_{1}\right)}\right]}
$$

\section{Setting the riskiness of a plan}

\subsection{Enumerative plans}

Risk is accounted for in sequential sampling in a variety of ways. First, consider the enumerative plan. As seen in eqns (4, 5, 8, \& 9), Kuno's and Green's plans require precision to be specified. In both these instances we chose to describe it in terms of the ratio of the sample standard error to the sample mean. Note that high $D$-values correspond to low levels of precision, and vice versa. The choice of the nominal precision level is arbitrary. Many have considered a $D$-value of 0.2 to 0.3 to be practicable and reasonable for pest sampling in routine monitoring programs, Hamilton and Hepworth [11]. A $D$-value of 0.25 enables detection of either a halving or a doubling of the sample mean.

\subsection{Binomial plans}

Risk is attended to in binomial plans by setting the type I and II error rates ( $\alpha$ and $\beta$ respectively) and the lower and upper bounds about the AT ( $\theta_{0}$ and $\theta_{1}$ respectively). The probability that a plan will suggest implementing control when it is not required (i.e. when $\theta \leq \mathrm{AT}$ ) is represented by $\alpha$, and $\beta$ is the probability that no control is recommended when it is in fact needed (i.e. when $\theta$ $>\mathrm{AT})$. The choice of error rates is subjective, although 0.1 has often been used for both $\alpha$ and $\beta$, Binns [12], Burkness et al. [13]. Others have argued that in a pest control context $\beta$ error is more serious (from the perspective of the primary producer at least), and thus should be set at a more conservative level than $\alpha$, Hamilton et al. [14], Mo and Baker [15]. Increases in either $\alpha$ or $\beta$ will see a concomitant increase in the probability of either an action or no action decision and a decrease in the likelihood of a no decision outcome.

Risk is accounted for in a more indirect way in binomial plans by setting the width of the region of indifference, i.e. the distance between $\theta_{0}$ and $\theta_{1}$. This region about the AT (i.e. the width of the AT band) effectively represents the emphasis or importance one wishes to place on the exact value of the AT. The derivation of ATs is often highly subjective and it may therefore be reasonable to apply some latitude. Within the region of indifference we are not too concerned as to whether or not the crop is treated. As with the error rates, setting $\theta_{0}$ and $\theta_{1}$ is a somewhat subjective task. Where the sampling unit is a plant, it may be reasonable to set the region as 5\% plants infested either side of AT [15]. 


\section{Assessing the achieved riskiness of a plan}

\subsection{Enumerative plans}

As described earlier, enumerative sequential sampling plans require precision to be set. It cannot be assumed, however, that this nominal precision level will actually be realised upon implementation of the plan. A useful way of assessing the achieved precision of a sampling plan is to statistically re-sample data-sets, Narajo and Hutchinson [16], Binns et al. [17]. We applied this approach to a $D$ $=0.3$ sampling plan for eggs of Helicoverpa spp. in fresh-market tomatoes (1,000 re-sampling iterations). The achieved levels of precision varied substantially (fig. 3). The mean achieved $D$ across all data-sets (i.e. mean of all means of 1,000 iterations) was 0.32 (SD 0.14 ), with the minimum and maximum being 0.22 (SD 0.27) and 0.46 (SD 0.21) respectively. In general, the plan failed to satisfy the nominal precision at densities of less than $0.2 \mathrm{eggs} /$ plant, whereas it often over-performed at higher densities. In this example the nominal and actual precision levels were close, on average. But this may not always be the case, and validation of precision is a prudent step that should be undertaken in the development of a sampling plan.

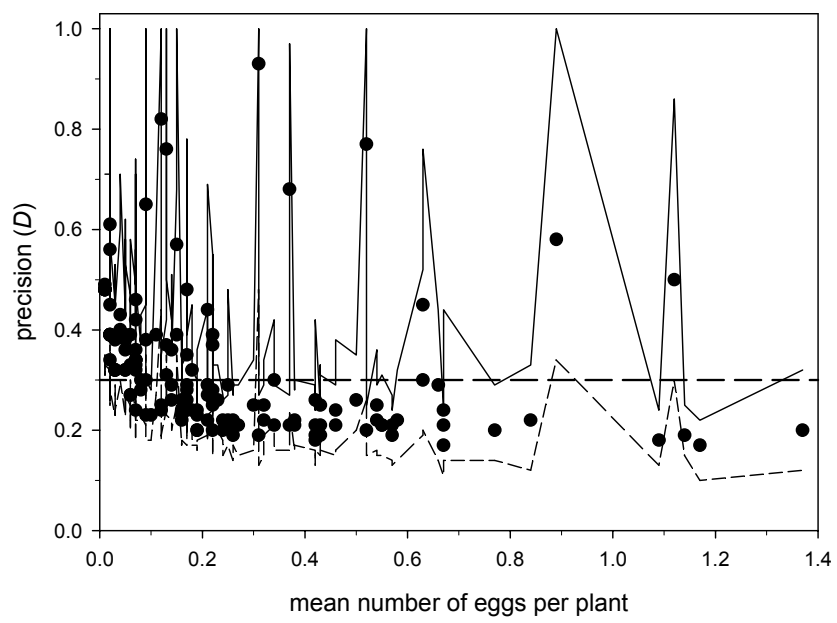

Figure 2: The mean (dots), minimum (light dashed line), and maximum (solid line) levels of precision $(D)$ achieved over 1,000 re-sampling iterations when implementing a sampling plan for Helicoverpa on fresh-market tomatoes. The heavy dashed line denotes the nominal level of precision $(D=0.3)$. Based on unpublished data of J.D. \& C.M. 


\subsection{Binomial plans}

The performance of binomial plans is typically assessed through the construction of operating characteristic (OC) and average sample number (ASN) curves. The OC function describes the probability that the plan will not recommend control, and the ASN function calculates the mean number of samples required to satisfy the sequential sampling stop rule. The OC of a perfect plan would be a stepwise function, as one would go from being $100 \%$ certain of the need to take action when above the AT (even if only by an infinitesimally small amount) to being $100 \%$ certain that no action is required when the level of infestation is at or below the AT. Thus, the steepness of the OC curve indicates the relative riskiness of the plan, where a steep slope implies a high-precision (and thus low risk) plan. Hamilton et al. [14] used the slope parameter of a four-parameter sigmoidal model (fitted to OC data generated by re-sampling-see below) as the criterion for comparing the relative precision of plans. The OC function can also be used to assess the level of conservativeness of a plan. If the $\mathrm{OC}$ is $<0.5$ at the $\mathrm{AT}$ then the plan is conservative, since at the AT it is more likely to recommend treatment than no treatment. Conversely, it is anti-conservative if the $\mathrm{OC}>0.5$ at the AT. The ASN curve is also useful from a risk management perspective in that it enables one to assess the likelihood of having to collect large sample sizes, which is often an important logistical consideration.

The OC function $(L(\theta))$ can be calculated according to Wald's [10] theoretical equations, where:

$$
L(\theta)=\frac{[(1-\beta) / \alpha]^{h(\theta)}-1}{[(1-\beta) / \alpha]^{h(\theta)}-[\beta /(1-\alpha)]^{h(\theta)}}
$$

where $\alpha$ and $\beta$ are the type I and II error rates, respectively, $\theta$ is a random variable of the population, which in the case of the binomial distribution (i.e. the distribution we are concerned with here) will represent the proportion of plants infested, and $h(\theta)$ is the non-zero solution for

$$
\int_{-\infty}^{\infty}\left[\frac{f\left(x, \theta_{1}\right)}{f\left(x, \theta_{2}\right)}\right]^{h(\theta)} f(x, \theta) d x=1 \quad \sum_{x}\left[\frac{f\left(x, \theta_{1}\right)}{f\left(x, \theta_{2}\right)}\right]^{h(\theta)} f(x, \theta)=1
$$

when $x$ is continuous (eqn 14) or discrete (eqn 15). Fowler and Lynch [18] provide a useful iterative procedure for computing OC values whereby dummyvalues are set for $h(\theta)$. They also explain how to attend to the special case where $h(\theta)=0$ (for OC and ASN curves). The ASN function, $E_{\theta}(n)$, is calculated as follows:

$$
E_{\theta}(n)=\frac{[\beta /(1-\alpha) L(\theta)]+(1-\beta) / \alpha[1-L(\theta)]}{\theta \cdot \ln \left[\frac{\theta_{1}\left(1-\theta_{0}\right)}{\theta_{0}\left(1-\theta_{1}\right)}\right]+\ln \left[\frac{1-\theta_{1}}{1-\theta_{0}}\right]}
$$


Construction of OC and ASN curves using Wald's algorithms is tedious, and it assumes that the population can be adequately described by a statistical distribution and that the sampling process can also be explained by a theoretical distribution. An alternative approach, developed by Naranjo and Hutchison [16], is to simulate sampling by re-sampling data-sets. In this instance, the OC is more specifically defined as the proportion of re-sampling iterations for which the proportion of plants infested did not exceed the lower of the two sequential stop lines. Similarly, the ASN represents the average number of samples (over all re-sampling iterations) that were collected before the stop-rule terminated sampling. Wald's OC and ASN algorithms are approximations as they do not account for overshooting of the decision boundaries. Re-sampling circumvents this problem, and it can accommodate for a minimum sample size that must be collected each time - there are at present no published adjustments to Wald's equations that do this. A potentially significant pragmatic constraint of the resampling method is that two independent data-sets are required, one to construct and one to validate the plan. Also, the number and spread of data-points is important, so as to enable the fit of a sensible model.

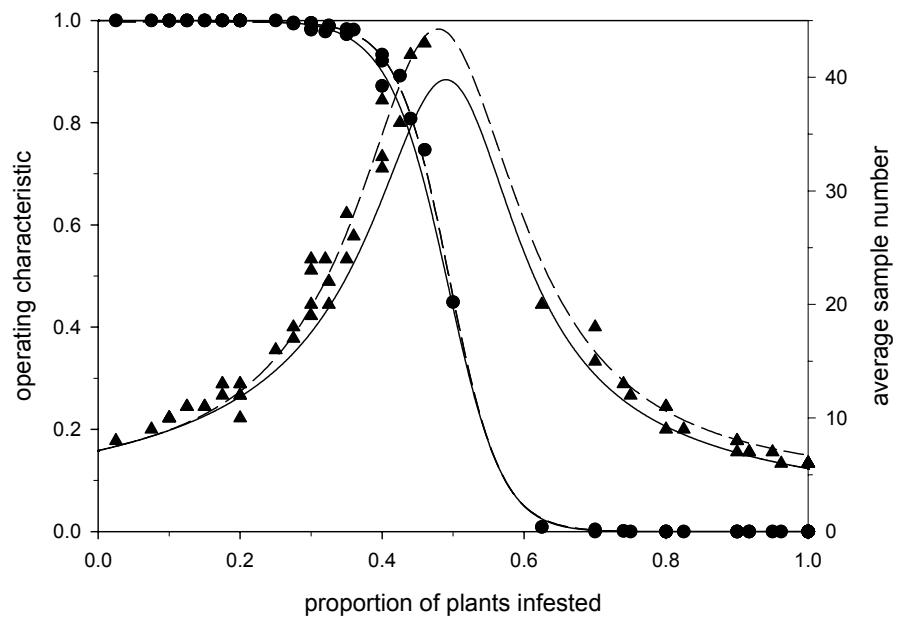

Figure 3: $\quad$ OC and ASN curves caluclated using Wald's algorithms (solid lines) and re-sampling (dashed). The specifications of the plan are: $\alpha=0.1$, $\beta=0.05, \theta_{0}=0.4$ and $\theta_{1}=0.6$. Dots and triangles represent the operating characteristic and mean sample size values respectively, calculated over 1,000 iterations, for data-sets of $P$. xylostella counts in broccoli fields (see [14]).

We determined $\mathrm{OC}$ and ASN curves for a binomial sampling plan for $P$. xylostella in broccoli crops (fig. 3). Theoretical and re-sampling approaches were used. The re-sampling-derived OC and ASN curves were obtained by fitting four-parameter sigmoidal and five-parameter Lorentzian models, 
respectively, to the resampled data using SigmaPlot [19]. The models respectively explained $>99.9 \%$ and $97.3 \%$ of the variance). The OC curves derived via theory and re-sampling were highly similar. Both implied a slightly conservative plan. There was a marked disparity between the ASN curves though, particularly around the AT (ASN at AT = 39.4 and 43.4), although such differences are unlikely to be of practical significance in this situation.

\section{Conclusion}

Sequential sampling offers a practical alternative to traditional high-power designs, and has particular applications in situations where phenomena are monitored on a routine basis and finances or time are limiting. The premise of sequential sampling is that the minimum possible number of samples should be collected each time a population is surveyed. As sample size decreases, however, confidence in our estimates of population parameters decreases, which plainly leads to an increase in the risk of making an inappropriate management decision. Therefore, the level of risk one is prepared to take needs to be stated and incorporated into the sampling plan, which then needs to be validated to determine how well it performed with respect to the risk level we specified. Risk is attended to enumerative plans through the concept of precision, and this can be assessed via re-sampling methods. For binomial plans, risk is incorporated through type I and II error rates and the upper and lower bounds of the AT. The performance of the plan can then be assessed through the construction of OC and ASN curves, which can be calculated theoretically or empirically.

\section{References}

[1] Hepworth, G. \& McFarlane, J. R., Variance of the estimated population density from a presence-absence threshold sample. Journal of Economic Entomology, 85(6), pp. 2240-2245, 1992a.

[2] Hepworth, G. \& McFarlane, J. R., A systematic presence-absence sampling method applied to twospotted mite, Tetranychus utricae Koch (Acari: Tetranychidae) on strawberries in Victoria, Australia. Journal of Economic Entomology, 85(6), pp. 2234-2239, 1992b.

[3] Karandinos, M.G., Optimal sample size and comments on some published formulae. Bulletin of the Entomological Society of America, 22(3), pp. 417-421, 1976.

[4] Kuno, E., A new method of sequential sampling to obtain population estimates with a fixed level of precision. Researches in Population Ecology, 11: pp. 127-136, 1969.

[5] Green RH., On fixed precision level sequential sampling. Researches in Population Ecology, 12, pp. 249-251, 1970.

[6] Iwao, S., A new regression method for analyzing the aggregation pattern of animal populations. Researches in Population Ecology, 10, pp. 1-20, 1968. 
[7] Lloyd, M., Mean Crowding. Journal of Animal Ecology, 36(1), pp. 1-30, 1967.

[8] Iwao, S. \& Kuno, E., Use of regression of mean crowding on mean density for estimating sample size and the transformation of data for the analysis of variance. Researches in Population Ecology, 10, pp. 210-214, 1968.

[9] Taylor, L.R., Aggregation, variance and the mean. Nature, 189(4766), pp. 732-735, 1961.

[10] Wald, A., Sequential analysis, John Wiley and Sons: Dover, NY, 1947.

[11] Hamilton, A.J. \& Hepworth, G., Accounting for cluster sampling in constructing enumerative sequential sampling plans. Journal of Economic Entomology, 97(3), pp. 1132-1136, 2004.

[12] Binns, M. R. Sequential sampling for classifying pest status (Chapter 8). CRC Handbook of Sampling Methods for Arthropods in Agriculture, eds. L.P. Pedigo \& G.D. Buntin,CRC Press: Boca Raton, pp.137-174, 1994.

[13] Burkness, E. C., Venette, R. C., O'Rourke, P. K., \& Hutchinson, W. D., Binomial sequential sampling for management of aster Leafhopper (Homoptera: Cicadellidae) and Aster Yellows phytoplasma in Carrot: impact of Tally Threshold on the accuracy of treatment decisions. Environmental Entomology, 28(5), pp. 851-857, 1999.

[14] Hamilton, A.J., Schellhorn, N.A., Ridland, P.M., Endersby, N.E. \& Ward, S.A., A dynamic binomial sequential sampling plan for diamondback moth, Plutella xylostella. Journal of Economic Entomology, 97(1), pp. 127-135, 2004.

[15] Mo, J. \& Baker, G., Evaluation of sequential presence-absence sampling plans for diamondback moth (Plutellidae: Lepidoptera) in cruciferous crops in Australia. Journal of Economic Entomology, 97(3), pp. 1118 $1125,2004$.

[16] Naranjo, S. E. \& Hutchinson, W. D., Validation of arthropod sampling plans using a resampling approach: software and analysis. American Entomologist, Spring, pp. 48-57, 1997.

[17] Binns, M.R., Nyrop, J.P. \& van Der Werf, W., Resampling to evaluate the properties of sampling plans (Chapter 9). Sampling and monitoring in crop protection, CABI Publishing: Oxon and New York, pp. 205-226, 2000.

[18] Fowler, G. W., \& Lynch A. M., Sampling plans in insect pest management based on Wald's sequential probability ratio test. Environmental Entomology, 16(2), pp. 345-354, 1987.

[19] SigmaPlot 2002 for Windows Version 8.02a, Systat Software, Inc. 